\title{
EL PROCESO DEL DUELO DENTRO DEL NÚCLEO FAMILIAR POR LA MUERTE DE UN INFANTE
}

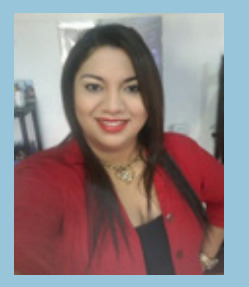

\section{Ingrid A. Benavides Hernández}

Dirección de Asistencia Social, Panamá

ingridbenavides8@gmail.com

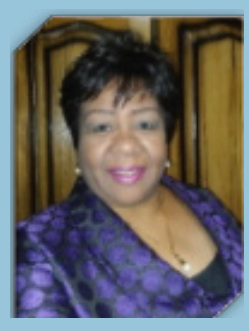

\section{Louisa Williams B.}

CRU de Bocas del Toro

Facultad de Ciencias de la Educación

Universidad de Panamá, Panamá

lbp.poket@gmail.com

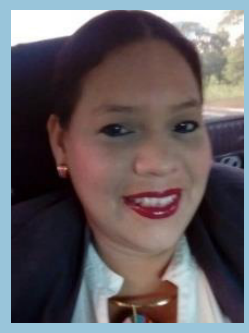

\section{Gissell Carrasquilla}

Trabajadora Social Especialista en Familia

Policía Nacional de Panamá

gicse2@yahoo.es

\section{RESUMEN}

Este ensayo presenta una forma clara de todo lo que sucede dentro del núcleo familiar cuando se pierde la vida de un Infante y describe los momentos difíciles que pasa la familia. El investigar sobre el duelo en este contexto, supone pieza clave para hacer comprender que perder un hijo sea posible uno de los dolores más grandes que la vida le puede dar y que al mismo tiempo ese proceso se convierte en uno de los duelos más largos y difíciles de comprender y de superar.

En esta investigación se recorre paso a paso cada etapa del duelo, cada sentimiento, cada momento que viven los miembros de una familia. Se indica además, que los duelos son manejados 
dentro de un núcleo familiar con el mismo proceso pero con distintos sentimientos, ya que según los estudios realizados, la madre puede sentir más dolor con la pérdida de un hijo que el padre; esto es así porque la madre es la que tiene una integración más fuerte con su hijo; lazos creados desde el vientre materno.

Se define y describe las etapas de: la negación, la ira, la negociación, la depresión y la aceptación manejadas dentro de una familia que lucha contra el dolor de la pérdida de un ser querido, al cual esperaron con amor y que por circunstancias de la vida perdieron. También se da a conocer que estos procesos de duelo tienen que ser muy bien manejados, porque causan en la familia un descontrol emocional muy fuerte que en ocasiones puede llegar a la ruptura del núcleo familiar, debido a que es posible que se pierda la comunicación durante el proceso del duelo, situación que puede culminar con la desintegración familiar. Finalmente, después del estudio las etapas, la familia se enfrenta a la realidad de que la vida continúa.

Palabras clave: Negación, Ira, Negociación, Depresión y Aceptación

\title{
THE GRIEVING PROCESS WITHIN THE FAMILY NUCLEUS DUE TO THE DEATH OF AN INFANT
}

\begin{abstract}
This essay presents a clear form of everything that happens within the family nucleus when the life of an Infant is lost and describes the difficult moments that the family goes through. The investigation of mourning in this context is a key piece to understand that losing a child is possible one of the greatest pain that life can give and that at the same time this process becomes one of the longest and difficult to understand and overcome.

This research describes, each stage of mourning, each feeling, every moment that the members of a family live, step by step. It is also indicated that mourning are handled within a family nucleus with the same process but with different feelings, since according to the studies performed, the mother may feel more pain with the loss of a child than the father; this is because the mother is the one who has a stronger connection with her son; ties created from the maternal womb.
\end{abstract}

It defines and describes the stages of: denial, anger, negotiation, depression and acceptance managed within a family that fights against the pain of the loss of a loved one, who are waited with love and that by circumstances of the life, became lost. It is also known that these processes 
of mourning have to be very well managed, because they can cause in the family a very strong emotional unrest that in times can reach the rupture of the family nucleus, because it is possible to lose the communication during the process of the mourning, situation that can culminate with the family disintegration. Finally, after studying the stages, the family faces the reality that life continues...

Key words: Negation (denial), Anger, Negotiation, Depression and Acceptance

\section{INTRODUCCIÓN}

El duelo es un proceso de adaptación emocional que sigue a la pérdida de un infante, familiar o amigo, ya que dentro del seno familiar, la pérdida de un hijo es una de las situaciones más difíciles de superar, sin importar la edad que tengan los hijos.

Al recabar información pertinente a este escrito, surge una gama diversa de tópicos en torno al duelo. No obstante, interesa en esta ocasión particular el duelo con la muerte de un Infante, específicamente en esta etapa de la vida y cómo superarlo, situación que me ha motivado a escribir este artículo.

Para los padres de familia la emoción que involucra procrear, el nacimiento y crianza de un hijo, es uno de los sentimientos más grandes y hermosos que experimenta el ser humano cuando logra hacer su vida mediante la formación de una familia.

Pero es muy difícil cuando en el entorno familiar, se pierde la vida de un infante, ya que la familia cae en un descontrol emocional único, porque el proceso del duelo toma el control total de la familia, este ensayo pretende dar a conocer las facetas por las cuales atraviesan, específicamente los padres, al perder a un hijo/a, a temprana edad; por lo que se ha estructurado el escrito con las siguientes secciones: negación, ira, negociación, depresión y aceptación dentro del proceso del duelo.

\section{La Negación}

Es importante entender que, cuando una persona pierde físicamente a otra, es muy probable que en ella se manifestarán diferentes reacciones emocionales y conductuales. Estas manifestaciones 
dependerán en diferentes variables como las creencias, cultura, situación económica, el vínculo familiar y hasta el nivel de educación de esta familia.

Lo que si es cierto es que entre más fuerte sea el vínculo familiar, mayor probabilidad que la reacción conductual o emocional sea más fuerte. Entre estas reacciones la más conocida en el proceso de un duelo es la Negación, como bien lo plantea Bautista (s/f) “... la negación es una defensa temporal para el individuo, y permite amortiguar el dolor ante una noticia inesperada e impresionante” (p. 24). Y para Ortego, López, Álvarez y Aparicio (2011) “...un primer momento de aturdimiento, "embotamiento", incredulidad, negación, shock o gran impacto emocional, en el que la persona puede llegar a poner en duda o no aceptar la triste realidad" (Ortego, et, al, 2011, p. 3).

Este tipo de reacción esta descrita generalmente en "la Teoría de Sigmund Freud, conocido como Mecanismos de Defensa" la cual nos dice que la Negación es un mecanismo de defensa que utiliza el ser humano para evadir responsabilidades y así poder bajar la carga de estrés; de manera inconsciente los familiares utilizan este método para poder así evitar pasar el dolor del duelo, por lo que se considera que ésta etapa es una de las más difíciles porque es en donde está más fuerte el dolor en la familia. Es en este sentido que Bautista (s/f) agrega que (...) "La muerte del hijo/a es considerada una de las situaciones más estresantes por las que puede pasar una persona ya que es una experiencia emocionalmente devastadora para los padres, independientemente de la edad de su hijo/a", (Bautista, s/f, p.23), lo que permite entender que las personas, los padres, pueden reaccionar de manera muy especial, al no aceptar la muerte de un hijo/a.

Esta fase de negación, provoca distintos comportamientos (que varían de persona en persona) como consecuencias del dolor ante la pérdida, los cuales, en ocasiones produce un alejamiento de la realidad. Así como lo expresa Díaz (2012)... "Negamos el hecho de la muerte, negamos la posibilidad de que no tengamos nunca más la posibilidad de estar con el ser querido, negamos que en algún momento podamos recuperarnos de esta (Díaz, 2012, p.16).

\section{La ira}

El privilegio de la concepción de una nueva vida, aquella que disfrutan los padres, se refleja en meses de alegría, cuidados, y ansiedad por conocer al nuevo ser que se espera habrá de nacer... y en la mayoría de los casos se convierte en una preparación de varios meses para el recibimiento y acomodo de ese bebe... y así en un estudio reciente, lo describe Oviedo, Urdaneta, Parra \& 
Marquina (2009)...”El nacimiento de un hijo, representa un momento estelar en la vida de una familia. Desde el embarazo hasta el parto, los padres desarrollan sentimientos de esperanza, ilusión y júbilo..." (Oviedo et al, 2009, p. 215); pero, ¿qué sucedería si por cualquier circunstancias de la vida, tempranamente se pierde? ¿Cómo reaccionaría los padres?, la negación da paso a la ira.

La ira en el duelo de un hijo según la tanatologa Elisabeth Kubler-Ross indica que durante este proceso es posible que se sienta enojo debido al inmenso dolor que da la partida de un ser querido. Puede ser que no estés enojado con ninguna persona, pero la incomprensión frente a la perdida de tu hijo, por ese dolor que se cree no merecer, hace que la persona quiera gritar toda la frustración que siente dentro del pecho.

La madre que pierde un hijo o una hija a muy temprana edad, se culpa a si misma por cuanto considera que debió de haberlo cuidado, que debió de haberlo protegido más, como lo afirman Díaz y Rolla (2006)...’Las madres en duelo sienten que han fallado en su función de protectoras... y la culpa se manifiesta no solo en el sentimiento de haber fallado en su función sino también de haber fallado al propio hijo/a...de haberlo desamparado..." (Díaz y Rolla, 2006, Citado por Geymonat, (2016, p.11).

Agobiados por la ira, los padres empiezan a buscar culpables o a reclamar ¿por qué a mí? ¿Por qué Dios me hizo esto?, empiezan las preguntas porque no pudieron salvarle la vida... miles de preguntas sin respuestas... bien arrojadas por esa ira... y esa impotencia... ¿qué pude haber hecho para salvarlo? Es en esta etapa de la ira es donde según Bautista (s/f), “...surgen todos los "porqué". Es una fase difícil de afrontar para los padres y todos los que los rodean. (Bautista, s/f, p.24).

Aquí el problema familiar se acrecienta más cuando uno de los miembros de la familia desvía injustamente esa ira que siente, a quienes lo rodean provocando conflictos familiares, y hasta provocando problemas físicos y emocionales como abuso de la comida, presión arterial, ansiedad, consumos de alcohol y drogas llevando a la familia que ya estaba afectada por el duelo de un hijo a confrontar ahora problemas nuevos dentro del núcleo familiar.

Estudios realizados han demostrado que la madre puede inclusive sentir mayor dolor y llevar el duelo de manera diferente a como lo haría el padre, debido a su cercanía e integración que mantiene con su hijo/a desde el vientre materno, en tal sentido Ewton DS, (1993) refiere que..."la madre en la muerte perinatal de su hijo puede crear una respuesta más severa e intensa que cuando ocurre la muerte de un familiar adulto..." (Ewton DS, 1993, citado por Oviedo, et al, 2009, p. 216). 
De igual manera, atendiendo a Geymonat (2016), “cada madre afrontará y elaborará el duelo de manera diferente, puesto que dicho proceso es absolutamente personal, el cual depende, entre otros factores, de las experiencias previas de duelo y el vínculo que unía madre-hijo”. (p.12).

\section{La Negociación: ¡también los niños mueren!}

Cronológicamente hablando en la vida familiar es lógico que primero mueren los padres y luego los hijos. Es conforme a Geymonat (2016)..."la ley natural indica que son los hijos quienes deben despedir y dar sepultura a sus padres", (p. 2), por eso es más difícil afrontar un proceso de duelo cuando este esquema es roto por las circunstancias de la vida; y ese pequeño ser que apenas empezaba una vivir no logra seguir más en este mundo porque le tocó padecer una enfermad, accidente o muerte repentina sin explicación alguna.

Para una madre y un padre ésta pérdida es considerada irreparable y muy difícil de sobre llevar. No obstante, es una carga muy pesada el continuar ahogándose en el dolor y se lucha por encontrar otras alternativas al dolor; a este proceso de negociación nos ayuda a entender al doliente en las palabras de Bautista (S/F) que explica "ante la dificultad de afrontar la difícil realidad, más el enojo con la gente y/o con Dios, surge la fase de intentar llegar a un acuerdo para intentar superar la traumática vivencia" (Bautista, S/A, p. 24). Ese acuerdo es entender que los niños también mueren... es el entender, según Geymonat (2016) que..."la muerte ha sido siempre motivo de incertidumbre que convoca al ser humano; única especie que reflexiona sobre su inexorable destino" (p. 6).

En la etapa de la negociación, empezamos a enfocarnos en pensar qué hubiéramos hecho de una manera distinta para poder evitar lo sucedido y también se nos viene a la mente las cosas que ya no podremos hacer más...la ira debe quedar atrás para poder aceptar la realidad de la pérdida. Nos ayuda en este aspecto Díaz (2012) cuando argumenta que la negociación es una forma de aceptación..."Es salir de la sensación de dolor, de temor, de incapacidad, de inseguridad, de pérdida junto con todos los temores y creencias limitantes que implica y, retomar, otra vez nuestra vida” (Díaz, 2012, p.14) agrega también “...llegamos a un compromiso con nosotros mismos y con el mundo", (Díaz, 2012, p.16). Este es el estadio necesario que propicia el salir del caos que produce el haber perdido al ser querido, en este caso a un infante. 


\section{La Drepresión}

Según la Organización Mundial de la Salud, la depresión es un trastorno mental que afecta muchas personas y puede convertirse en un grave problema dependiendo a su intensidad. La depresión por la pérdida de un hijo en el núcleo familiar, es agobiante. Aunado a la incapacidad a la impotencia de comprender y procesar el sufrimiento que embarga a los dolientes, provoca toda clase de alteraciones que obviamente pueden afectar la salud. Tanto es así que según Bautista (s/f), “...cuando no se puede seguir negando, la persona se debilita, adelgaza, aparecen otros síntomas y se verá invadida por una profunda tristeza" (Bautista s/f, p. 24). En este estadio conocido como depresión, las parejas dolientes pueden confrontar conflictos y pueden sentir que no son atendidos ni comprendidos el uno al otro, lo que puede acarrear más complicaciones; sustenta Díaz (2012)... "todos somos diferentes y hay personas a las que les cuesta más tiempo que a otras procesar su duelo" (Díaz, 2012, p. 17); es aquí donde aflora la capacidad individual de manejar el duelo o dejarse consumir por el dolor.

La depresión por duelo se refleja hasta en la manera de vestirse: ropa sobria o totalmente oscura (negro), en ocasiones vestirse de blanco...; dejar de hacer las cosas que era de su agrado y abstenerse de interactuar con otras personas y en este caso específico, el compartir con amistades o familiares que tienen hijos de la edad del hijo que ha fallecido. Se desconoce una fecha tope para que esta fase culmine pero según Bosquet dice: “...la pérdida de un hijo supone un evento traumático "...por lo que no es de extrañar que un $20 \%$ de los padres no lleguen a superarlo nunca." (Bosquet, et al, 2010, p.65, Citado por Geymonat, 2016, p.3).

Para salir de una fase depresiva se requiere una disposición física, mental y tal vez ayuda de profesionales o quizás de medicamentos especiales. Una disposición física y mental requiere de la madurez de los dolientes, es aprender a manejar el duelo... así como lo expresa Díaz (2012)..."El manejo del duelo es aceptar la pérdida como una realidad..." el proceso de duelo debe inducir a la persona a entender finalmente que lo que le acontece es real y debe aceptarlo. (Díaz, 2012, p.14).

\section{La fase de aceptación: La vida continúa.}

La muerte de un hijo obliga a cambiar toda la dinámica de la vida que involucra el entorno familiar. Todo cambia y esto demanda el aceptar que la vida ya no va ser igual que antes. La aceptación es en este momento del duelo donde ya se percibe un sentimiento de paz; en donde la rutina diaria comienza a ser parte de la vida. 
Se pasa a entender finalmente la realidad... que ese hijo que tanto se esperó por nacer y verlo crecer y al que se amó ya no estará más. Es aquí en donde los padres comienzan a analizar y ver desde otro punto de vista las cosas... se empieza ya a recordar todos los momentos felices que compartieron con ese hijo/a; la familia comienza nuevamente a lograr seguir adelante sin un miembros más que en algún momento fue el complemento de su felicidad.

La familia que comparte este sufrimiento, observa y es parte del proceso y también espera poder superarlo. No obstante, para que eso suceda, deben permitir que a través del duelo, los padres experimenten el dolor que surge de la perdida. Solo así, sanando ellos primero, todos puedan volver a la realidad y continuar con sus vidas. Esto no implica que deben olvidar sino... aceptar y continuar.

La aceptación de que la vida continua no es fácil, requiere del amor y compromiso entre los padres y de las personas que rodean. Algunos padres, especialmente las madres refieren que jamás han olvidado a ese hijo/a que han perdido a la muerte que en ocasiones vuelve el dolor, a este respecto tenemos que según Bautista:

“(... con el tiempo...) a pesar de todo, nada vuelve a ser como antes, no se recobra la mente pre duelo, aunque sí parece llegarse, con el tiempo, a un duelo latente, más suave y menos doloroso, que se puede reactivar en cualquier momento ante estímulos que recuerden. (Bautista, s/f, p.25).

La aceptación implica entre otras cosas algunas interrogantes: ¿Debemos olvidar? ¿Es fácil olvidar? El quedarse debatiendo entre estas ideas no conduce a superar el dolor y es que la vida continúa y también continúa para el doliente.

\section{CONCLUSIONES}

Los estudiosos enmarcan el proceso de duelo con las siguientes fases: negación, ira, negociación, depresión y aceptación. Es posible que otros autores identifiquen otras facetas.

El nacimiento de un hijo es un regalo de Dios, el tener un hijo es un privilegio. Sin embargo, la vida tiene sus enigmas y se puede llegar a perder lo que tanto se ama.

El duelo por el fallecimiento de un infante es altamente traumático para los padres y en 
ocasiones desbastador; pueden inclusive no superarlo jamás. Los padres y mayormente las madres (por el nexo desde su vientre materno) llegan a culparse constantemente pensando que no fueron capaces de protegerlos y se continúan preguntando en dónde fallaron.

Por ley natural se cree que son los hijos los que deben padecer el duelo por la pérdida de sus padres no los padres por los hijos.

Las personas manejan el duelo de manera diferente y esto puede ser fundamental para la recuperación o la prolongación del dolor ante la pérdida. Es importante que los dolientes ante una circunstancia tan adversas como la muerte de un niño/a, procesen su duelo y que al final recuperen la sanidad mental y afronten la vida que tienen por delante.

En todo caso, el manejar el duelo apropiadamente o no, es una decisión personal. Sin embargo, se aboga a que se oriente a los dolientes a permitir que retomen sus vidas... sin olvidar, pero a continuar viviendo.

\section{BIBLIOGRAFÍA}

Bautista, P. (s/f). El duelo ante la muerte de un recién nacido. Neuquén, Argentina: Servicio de Neonatología del Hospital Castro Rendón, Enfermería Neonatal 23,

Díaz, E. (2012). El duelo y su proceso para superarlo. A.C, México, D.F: Asociación Mexicana de Tanatología.

Geymonat, N. (2016). Duelo en madres que han perdido un hijo de manera inesperada. Montevideo, Uruguay: Universidad de la República Facultad de Psicología.

Ortego, M., López, s., Álvarez, M. y Aparicio M. (2011). El duelo. España: Universidad de Cantabria, Departamento de Enfermería.

Oviedo, S., Urdaneta, E., Parra, F., Marquina, M. (2009). Duelo materno por muerte perinatal. México, D.F: Revista Mexicana de Pediatría Vol. 76, Núm. 5 - Septiembre-Octubre 2009 Pág. 215219.

UMECIT. (2017). Seminario Taller: Redacción de Artículos Científicos. Chitré - Panamá: Universidad Metropolitana de Educación, Ciencias y Tecnología. 8-9 de julio. 